\title{
Compliance of the cerebrospinal space: comparison of three methods
}

\author{
Agnieszka Kazimierska $^{1}$ (D) Magdalena Kasprowicz ${ }^{1} \cdot$ Marek Czosnyka $^{2,3} \cdot$ Michał M. Placek $^{2} \cdot$ Olivier Baledent $^{4}$. \\ Peter Smielewski ${ }^{2} \cdot$ Zofia Czosnyka $^{2}$
}

Received: 3 December 2020 / Accepted: 26 March 2021 / Published online: 14 April 2021

(C) The Author(s) 2021

\begin{abstract}
Background Cerebrospinal compliance describes the ability of the cerebrospinal space to buffer changes in volume. Diminished compliance is associated with increased risk of potentially threatening increases in intracranial pressure (ICP) when changes in cerebrospinal volume occur. However, despite various methods of estimation proposed so far, compliance is seldom used in clinical practice. This study aimed to compare three measures of cerebrospinal compliance.

Methods ICP recordings from 36 normal-pressure hydrocephalus patients who underwent infusion tests with parallel recording of transcranial Doppler blood flow velocity were retrospectively analysed. Three methods were used to calculate compliance estimates during changes in the mean ICP induced by infusion of fluid into the cerebrospinal fluid space: (a) based on Marmarou's model of cerebrospinal fluid dynamics $\left(\mathrm{C}_{\mathrm{CSF}}\right)$, (b) based on the evaluation of changes in cerebral arterial blood volume $\left(\mathrm{C}_{\mathrm{CaBV}}\right)$, and (c) based on the amplitudes of peaks $\mathrm{P} 1$ and $\mathrm{P} 2$ of ICP pulse waveform $\left(\mathrm{C}_{\mathrm{P} 1 / \mathrm{P} 2}\right)$.

Results Increase in ICP caused a significant decrease in all compliance estimates $(p<0.0001)$. Time courses of compliance estimators were strongly positively correlated with each other (group-averaged Spearman correlation coefficients: 0.94 [0.880.97 ] for $\mathrm{C}_{\mathrm{CSF}}$ vs. $\mathrm{C}_{\mathrm{CaBV}}, 0.77$ [0.63-0.91] for $\mathrm{C}_{\mathrm{CSF}}$ vs. $\mathrm{C}_{\mathrm{P} 1 / \mathrm{P} 2}$, and 0.68 [0.48-0.91] for $\mathrm{C}_{\mathrm{CaBV}} \mathrm{vs.} \mathrm{C}_{\mathrm{P} 1 / \mathrm{P} 2}$ ).

Conclusions Indirect methods, $\mathrm{C}_{\mathrm{CaBV}}$ and $\mathrm{C}_{\mathrm{P} 1 / \mathrm{P} 2}$, allow for the assessment of relative changes in cerebrospinal compliance and produce results exhibiting good correlation with the direct method of volumetric manipulation. This opens the possibility of monitoring relative changes in compliance continuously.
\end{abstract}

Keywords Intracranial pressure $\cdot$ Cerebrospinal compliance $\cdot$ Infusion test $\cdot$ Cerebral arterial blood volume $\cdot$ Pulse waveform

\section{Introduction}

In adults, the skull is a closed non-distensible box filled with three volume components: brain tissue, cerebral blood, and

Agnieszka Kazimierska

agnieszka.kazimierska@pwr.edu.pl

1 Department of Biomedical Engineering, Faculty of Fundamental Problems of Technology, Wroclaw University of Science and Technology, Wroclaw, Poland

2 Brain Physics Laboratory, Division of Neurosurgery, Department of Clinical Neurosciences, Addenbrooke's Hospital, University of Cambridge, Cambridge, UK

3 Institute of Electronic Systems, Faculty of Electronics and Information Technology, Warsaw University of Technology, Warsaw, Poland

4 Department of Medical Image Processing, CHU Amiens, University of Picardy Jules Verne, Amiens, France cerebrospinal fluid (CSF) [16]. According to the MonroKellie doctrine, in the long-term-and under normal conditions - increases in the volume of one component are compensated by decreases in the volume of another, maintaining the total volume at an approximately constant level [20]. However, in the short-term scale, considering pulsatile blood flow, for a part of the cardiac cycle, total blood volume increases and then decreases, leaving total volume changes equal to zero [2]. This produces rhythmic instability of the intracranial pressure (ICP) signal, known as 'pulse waveform'. The ability of the cerebrospinal space to accommodate changes in volume is quantified by a parameter called compliance which links changes in volume with corresponding changes in pressure [23]. Low compliance puts the system at risk of disproportionately large increases in ICP for even small increases in intracranial volume, potentially leading to intracranial hypertension [21]. To measure cerebrospinal compliance, external gradual volume addition into the cerebrospinal space is needed, which is a limitation preventing continuous 
monitoring. We aimed to examine and compare three methods of assessment of cerebrospinal compliance. One is a 'golden standard' based on external volume load, and two others rely on the evaluation of changes related to blood stroke volume over heart period. These two, if they are linked to 'gold standard' compliance, would allow for continuous monitoring in various clinical scenarios, for instance, hydrocephalus ICP monitoring or traumatic head injury (TBI) and subarachnoid haemorrhage neuro-intensive care monitoring.

The infusion study is a type of volume-pressure test based on the injection of additional volume into the cerebrospinal space that allows for the estimation of compensatory parameters, including cerebrospinal compliance, from a mathematical model of CSF circulation [13]. In the present work, we compared compliance estimates obtained with the model of CSF dynamics (the 'gold standard' method) during changes in the mean ICP induced by constant rate infusion of fluid into the CSF space in normal-pressure hydrocephalus (NPH) patients with two other measures: based on the estimation of changes in cerebral blood volume (CBV) in each cardiac cycle and based on the analysis of changes in the ratio of characteristic peaks P1 and P2 of ICP pulse waveform. The study offers new insight into the feasibility of using the $\mathrm{P} 1 / \mathrm{P} 2$ ratio and evaluation of changes in $\mathrm{CBV}$ as a tool for continuous monitoring of compliance of the cerebrospinal space.

\section{Materials and methods}

\section{Data acquisition}

Data from patients who underwent infusion studies with simultaneous recording of ICP and cerebral blood flow velocity (CBFV) signals at Addenbrooke's Hospital (Cambridge, UK) between 1993 and 1998 were selected for retrospective analysis in this study. Selection of patients was made on a basis of good quality of ICP pulse waveform and CBFV recording, and only those tests where the shape of the pulse waveform of ICP presented both $\mathrm{P} 1$ and $\mathrm{P} 2$ peaks were classified as suitable for analysis. Out of the full group of 72 considered recordings, 36 were chosen. Sixteen recordings $(22 \%)$ were excluded because of rounded ICP pulse waveform that did not allow for the identification of both characteristic peaks, and 20 recordings $(28 \%)$ were excluded due to insufficient quality of either ICP or CBFV signals. The data were collected as part of routine clinical investigation following diagnosis of NPH. Ethics committee approval to record ICP and CBFV using transcranial Doppler (TCD) ultrasonography was obtained.

The computerized infusion test was performed with two hypodermic needles ( 25 gauges). One of the needles was used for ICP measurement and connected to a pressure transducer through a saline-filled tube. The second needle was used for infusion and connected to an infusion pump containing a pressure amplifier (Simonsen \& Will, Sidcup, UK). The infusion of normal saline into the CSF space was performed at the rate of $1.5 \mathrm{ml} / \mathrm{min}$. The test began with 10 min of baseline recording of ICP before the start of the infusion. Infusion continued until the increase in ICP reached either the plateau phase or the maximum acceptable level of $40 \mathrm{~mm} \mathrm{Hg}$, and the recordings were further collected until ICP returned to baseline level. CBFV in the middle cerebral artery was monitored during the test using a TCD unit (Neuroguard; MedaSonics, Fremont, CA, USA) with a 2-MHz probe locked in a stable position using a commercially available fixation system. Twenty-six recordings included also arterial blood pressure (ABP) monitored noninvasively using a photoplethysmographic system (Finapres; Finapres Medical Systems, the Netherlands).

An analogue-to-digital converter (DT 2814; Data Translation, Marlboro, USA) connected to an IBM AT laptop computer (Amstrad ALT 386 SX; Amstrad, Brentwood, UK) was used for the collection of data from the pressure monitors and the TCD system. The signals were sampled at frequency ranging from 30 to $50 \mathrm{~Hz}$ using custom software for waveform recording (WREC; W. Zabolotny, Warsaw University of Technology, Warsaw, Poland). An illustrative example of recorded signals is presented in Fig. 1.

\section{Three methods of estimation of cerebrospinal compliance}

The relationship between pressure and volume in the intracranial space is described by an exponential function known as the pressure-volume curve, which shows that changes in ICP increase progressively with the mean ICP [29]. Traditionally, measurement of the pressure response requires external manipulation of intracranial volume, such as bolus injection or constant rate infusion $[18,24]$. A different approach to compliance estimation relies on the possibility of measuring changes in CBV alongside ICP. However, a method based on magnetic resonance imaging [1], although potentially accurate, is currently only applicable to the evaluation of instantaneous, or 'snapshot', values, and continuous estimation of changes in $\mathrm{CBV}$ using $\mathrm{TCD}$ recordings of $\mathrm{CBFV}$ in large cerebral arteries [19] does not allow for calibration of obtained values due to the unknown cross-sectional area of insonated vessels.

It has also been suggested that information about cerebral compliance may be extracted from the pulse waveform of ICP itself [6]. Three major features commonly used to characterize ICP pulse waveform are peaks P1, P2, and P3 [16]. Although the precise origin of those peaks is not universally agreed upon, the shape of the ICP waveform is believed to arise from 

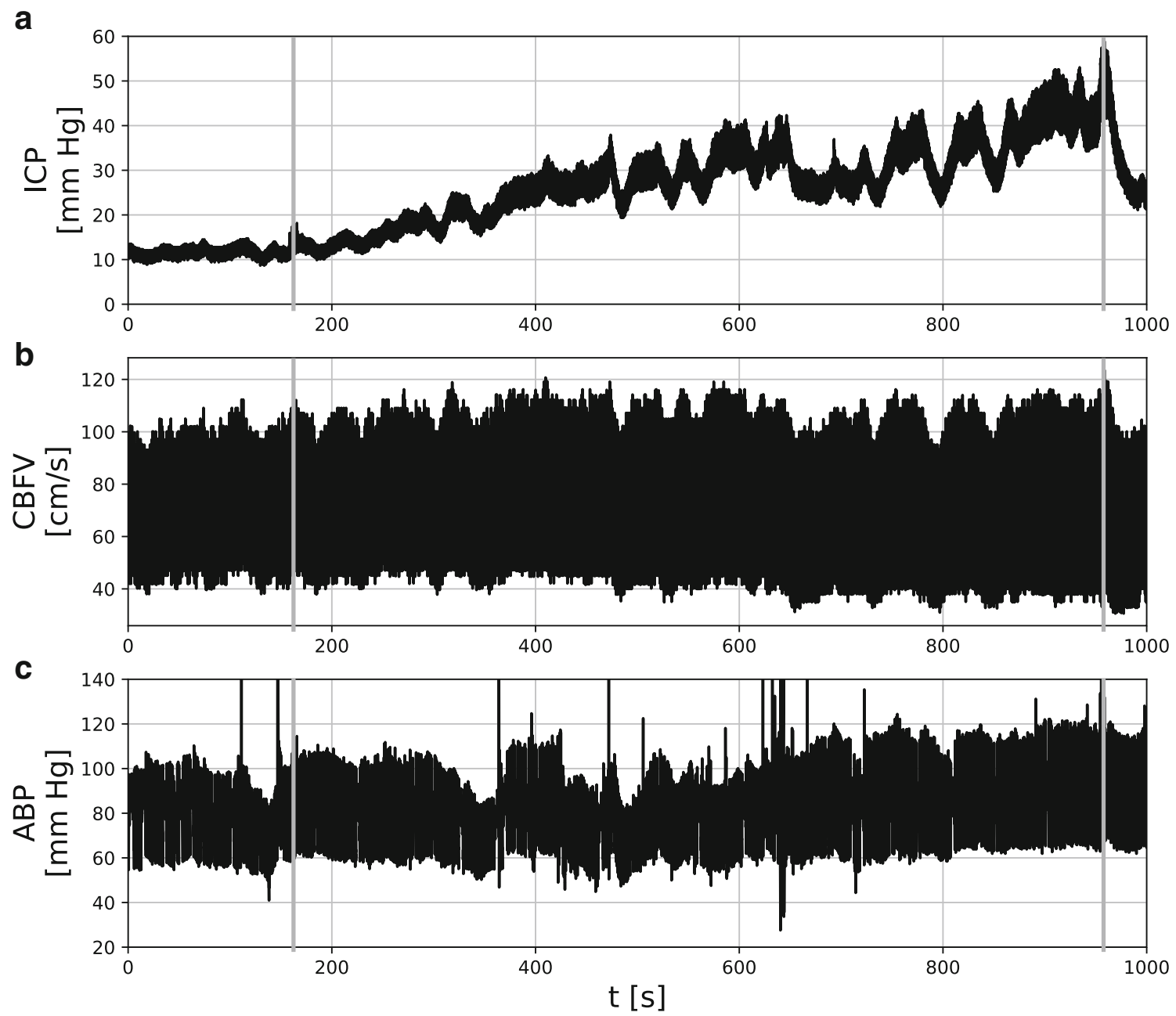

Fig. 1 Illustrative example of signals recorded during the infusion test for a single patient. Grey vertical lines indicate the start and end of constant rate $(1.5 \mathrm{ml} / \mathrm{min})$ infusion. a Intracranial pressure (ICP). b Cerebral blood flow velocity (CBFV). c Arterial blood pressure (ABP)

changes in both ABP and CBV [7]. Under normal circumstances, P1 dominates over the other two peaks, resulting in a saw-tooth appearance of the waveform (Fig. 2a). As the mean ICP increases, so does the amplitude of characteristic peaks. However, the change is not uniform, and rising prominence of P2 (Fig. 2b) eventually leads to a rounded or triangular waveform with indistinguishable P1 and P3 $[9,10]$. Given the relatively larger changes in the magnitude of the P2 component, it has been suggested that the ratio of peak amplitudes, P1/P2, may provide information about cerebral compliance. Still, despite its potential as a means for longterm monitoring of the state of the cerebrospinal space, very little attention has been devoted to the application of this parameter in the evaluation of cerebral compensatory reserve.

In the present study, three methods were used to obtain estimates of cerebrospinal compliance: (a) based on a model of CSF dynamics, (b) based on evaluation of changes in CBV, and (c) based on the ICP pulse waveform. Assessment of model-based parameters describing CSF dynamics (a) was performed using built-in algorithms of specialized software
(ICM+; Cambridge Enterprise, Cambridge, UK). All other analyses were performed using programmes custom-written in Python 3.8.

\section{Model of cerebrospinal fluid dynamics}

The first estimate of cerebrospinal compliance (denoted $\mathrm{C}_{\mathrm{CSF}}$ from 'cerebrospinal fluid dynamics') was obtained from a mathematical model of CSF volume-pressure compensation [12]. Under normal conditions, in the absence of long-term fluctuations in cerebral blood volume, it is assumed that the production of CSF is balanced by its storage and reabsorption. Including external CSF infusion, this can be described by the following relationship:

production of CSF + infusion

$$
=\text { storage of } \mathrm{CSF}+\text { reabsorption of } \mathrm{CSF}
$$

The rate of CSF production is assumed to be constant. $I(t)$ is the rate of the external volume infusion (e.g., $1.5 \mathrm{ml} / \mathrm{min}$ in 
a

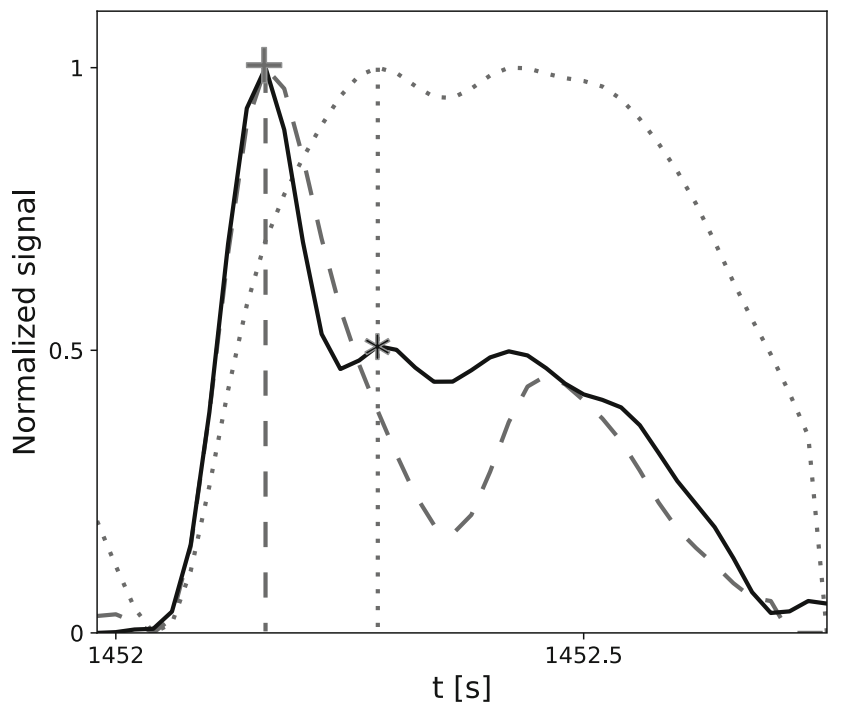

Fig. 2 Illustrative examples of intracranial pressure (ICP) pulse waveforms from two different patients. Location of peaks P1 and P2 is indicated by cross (P1) and star (P2) signs. ICP signals are plotted with solid black lines. Additional signals used in the process of peak detection are plotted as dashed (arterial blood pressure (ABP)) and dotted (cerebral

the present study). The rate of CSF storage $\left(I_{\mathrm{s}}\right)$ is proportional to cerebrospinal compliance $(C)$ and depends on the derivative of CSF pressure $(P)$ over time $(d P / d t)$ :

$I_{s}=C \frac{d P}{d t}$

Compliance is in turn inversely proportional to the gradient of CSF pressure and reference pressure $\left(P_{0}\right)$ :

$C=\frac{1}{E\left(P-P_{o}\right)}$

where $E$ is cerebral elasticity. The rate of CSF reabsorption $\left(I_{\mathrm{r}}\right)$ is proportional to the gradient between CSF pressure $P$ and pressure in the sagittal sinuses $\left(P_{\mathrm{ss}}\right)$ :

$I_{r}=\frac{P-P_{s s}}{R_{\text {out }}}$

where $R_{\text {out }}$ describes the resistance to CSF outflow. Relationship (3) is considered valid only above certain pressure level described as 'lower breakpoint pressure'. It is the pressure above which the cerebrospinal pressure-volume curve becomes exponential. Below this pressure, the pressure-volume relationship is linear. The combination of Eqs. (1) to (4) produces the final equation:

$\frac{1}{E\left(P-P_{0}\right)} \frac{d P}{d t}+\frac{P-P_{b}}{R_{\text {out }}}=I(t)$

where $P_{\mathrm{b}}$ is the baseline pressure. b

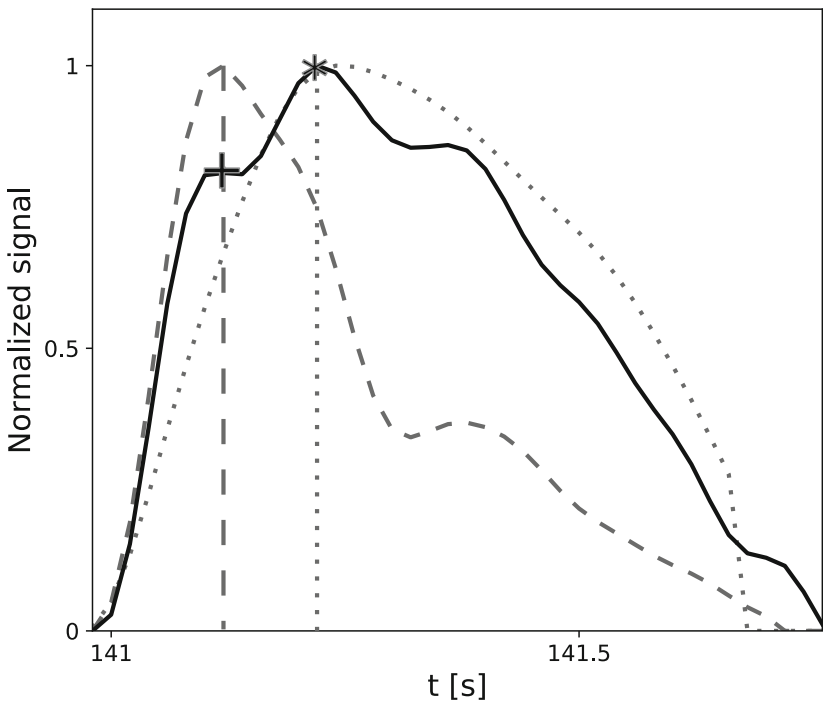

arterial blood volume (CaBV)) lines. All signals are normalized and aligned with regard to pulse onset location. Vertical lines indicate the correlation between the position of peaks $\mathrm{P} 1$ and $\mathrm{P} 2$ and the local maxima of the $\mathrm{ABP}$ (dashed line) and $\mathrm{CaBV}$ (dotted line) waveforms, respectively

Parameters $R_{\text {out }}, E$, and $P_{0}$ were estimated for ICP recordings used in this study based on the analytical solution of Eq. (5) for constant rate infusion. $E$ and $P_{0}$ were then used to obtain estimates of cerebrospinal compliance over the course of the infusion test from Eq. (3). To maintain cohesion with the other two pulse-by-pulse methods of estimation, the mean ICP over each cardiac cycle was taken as $P(t)$.

\section{Evaluation of pulsatile changes in cerebral blood volume}

The second estimate of cerebrospinal compliance (denoted $\mathrm{C}_{\mathrm{CaBV}}$ from 'cerebral arterial blood volume') was obtained from a mathematical model of cerebral blood circulation using the approach described by Kim et al. [19]. The change in CBV over a single cardiac cycle can be expressed as the difference between arterial inflow $\left(\mathrm{CBF}_{\mathrm{a}}\right)$ and venous outflow $\left(\mathrm{CBF}_{\mathrm{v}}\right)$ :

$\Delta C B V(t)=\int_{t_{0}}^{t}\left(C B F_{a}(s)-C B F_{v}(s)\right) d s$

where $t_{0}, t$ are the beginning and end of the cardiac cycle, respectively, and $s$ is the variable of integration. Given the assumption that venous outflow has low pulsatility compared to arterial inflow, the former can be approximated by a constant flow equal to averaged arterial inflow:

$\Delta C_{a} B V(t)=\int_{t_{0}}^{t}\left(C B F_{a}(s)-\right.$ mean $\left.C B F_{a}\right) d \mathrm{~s}$ 
Furthermore, cerebral arterial blood flow can be expressed as cerebral blood flow velocity multiplied by cross-sectional area of the vessel. Using TCD recordings of CBFV in cerebral arteries and assuming that the crosssectional area of insonated vessel remains constant, Eq. (7) can be approximated as

$$
\Delta C_{a} B V(n)=S_{a} \cdot \sum_{i=1}^{n}\left(C B F V_{a}(i)-\text { meanCBFV } V_{a}\right) \Delta t(i)
$$

where $n$ is the number of samples from the beginning of the cardiac cycle, $\Delta t$ is the time interval between two consecutive samples, and $S_{\mathrm{a}}$ is the unknown cross-sectional area of insonated vessel.

Based on pulsatile signals $\mathrm{CaBV}$ and ICP, cerebrospinal compliance $(C)$ can be estimated as

$C=\frac{A M P_{C_{a} B V}}{A M P_{I C P}}$

where $A M P_{\mathrm{CaBV}}$ and $A M P_{\mathrm{ICP}}$ are the amplitudes of fundamental components of $\mathrm{CaBV}$ and ICP, respectively. However, due to unknown $S_{\mathrm{a}}$, resulting values of $C$ cannot be calibrated in units of $\mathrm{ml}$ per $\mathrm{mm} \mathrm{Hg}$.

In this study, CaBV was estimated from Eq. (8) based on CBFV recordings in the middle cerebral artery. Peak-to-peak amplitudes of $\mathrm{CaBV}$ and ICP in each cardiac cycle were then used to obtain pulse-by-pulse estimates of cerebrospinal compliance using Eq. (9).

\section{ICP waveform analysis}

The third estimate of cerebrospinal compliance (denoted $\mathrm{C}_{\mathrm{P} 1 /}$ P2 from the P1/P2 amplitude ratio) was based on the analysis of the ICP pulse waveform. Prior to the analysis, ICP, CBFV, and $\mathrm{ABP}$ (where available) signals were filtered using a lowpass filter with the cut-off frequency of $10 \mathrm{~Hz}$. Individual pulses in the ICP signal were identified using a modified Scholkmann algorithm [4]; corresponding sections of CBFV and ABP signals were extracted based on pulse onset locations from ICP. For the purpose of peak annotation, each pulse was normalized and linearly detrended, and the three signals were aligned with regard to pulse onset in order to remove the phase shift resulting from distance between measurement sites.

A semi-automated algorithm based on the detection of the local maxima was used for peak identification. The algorithm incorporated information about the local maxima of $\mathrm{ABP}$ and $\mathrm{CaBV}$ (derived from the CBFV signal using Eq. (8)), taking into account observations from previous studies which showed that $\mathrm{P} 1$ is associated with the propagation of the pulse pressure wave through cerebral arteries and usually coincides with the systole of ABP, while P2 is derived from arterial blood volume load and coincides with the maximum of $\mathrm{CaBV}[8,15]$. In each pulse waveform, the local maxima of ICP corresponding to the position of maxima in $\mathrm{ABP}$ and $\mathrm{CaBV}$ were selected as candidates for $\mathrm{P} 1$ and $\mathrm{P} 2$, respectively. In the absence of the ABP signal, the first maximum of the CBFV signal was used instead to identify P1 candidates. Full detection results were reviewed and manually corrected in cases of insufficient detection accuracy, particularly in pathologically rounded waveforms. Pulses with distorted ICP waveform or unidentifiable P1 and P2 were excluded from further analyses. Illustrative examples of ICP pulse waveforms with peak annotations are presented in Fig. 2.

The amplitude of peaks P1 and P2 in each pulse was calculated as the vertical distance to the preceding local minimum identified as pulse onset. Pulse-by-pulse P1/P2 amplitude ratio was then used as a compliance estimate.

\section{Statistical analysis}

Statistical analyses were performed using Python 3.8 with the built-in methods included in the SciPy 1.5.0 package. The Shapiro-Wilk test with a significance level of 0.05 was used to assess normality of data distributions. Upon rejection of the normality hypothesis for most of the analysed variables, non-parametric methods were chosen to analyse the relationship between compliance estimates. Time courses of compliance estimates obtained for individual patients with each of the three methods were compared with each other and with ICP using the Spearman correlation coefficient. To reduce the effect of difference in time scales used in compliance estimation, 30-pulse moving averages were used.

In order to compare the 'high' and 'low' compliance states reflecting the baseline and plateau phase of the infusion test, 1-minute-long fragments of the recordings where all three compliance estimates could be obtained were selected manually. Due to significant distortion of the ICP pulse waveform during baseline in a number of recordings, the initial stage of infusion was selected instead as baseline. Two patients for whom baseline values were not available either due to low quality of the ICP signal or the lower breakpoint pressure limit used in estimation of $\mathrm{C}_{\mathrm{CSF}}$ were excluded from this part of the analysis. Values averaged over the baseline and plateau phases of the infusion test were compared using the Wilcoxon signed rank test. The same methods were used to determine the significance of changes in amplitude of peaks $\mathrm{P} 1$ and $\mathrm{P} 2$.

A significance level of 0.05 was assumed in all statistical tests. All group-averaged values are presented as median [first quartile-third quartile]. 


\section{Results}

\section{Patient characteristics}

The mean age of the patients was 54 years (range, 27-76 years). The patients showed ventricular dilation marked by increased bicaudate index (mean, 0.27; range, 0.14-0.39), and $14 \%$ showed white matter ischemia. Initial ICP in the group was 8.7 [3.8-11.4] $\mathrm{mm} \mathrm{Hg}$. Group-averaged $\mathrm{R}_{\text {out }}$ and elasticity were $12.1[8.9-15.8] \mathrm{mm} \mathrm{Hg} /(\mathrm{ml} / \mathrm{min})$ and 0.19 $[0.14-0.33] \mathrm{ml}^{-1}$, respectively.

\section{Amplitude of peaks P1 and P2 during changes in mean ICP}

Figure 3 shows an illustrative example of the time courses of P1 and P2 amplitude during infusion test for a single patient. The rise in the mean ICP during infusion resulted in an increase in amplitude of both P1 and P2 $\left(p<10^{-6}\right)$, with a visibly larger change for P2: up to 6.22 [4.44-8.35] mm Hg $\mathrm{mm} \mathrm{Hg}$ from baseline amplitude of 3.52 [2.44-4.49] $\mathrm{mm} \mathrm{Hg}$ vs. 4.23 [2.7-4.74] $\mathrm{mm} \mathrm{Hg}$ from baseline of 2.51 [1.44-3.56] $\mathrm{mm} \mathrm{Hg}$ for P1. While baseline P1/P2 ratio varied between patients, with some patients exhibiting pronounced $\mathrm{P} 1$ ( $\mathrm{P} 1 /$ $\mathrm{P} 2>1)$ and some already showing increased $\mathrm{P} 2(\mathrm{P} 1 / \mathrm{P} 2<$ 1 ), the $\mathrm{P} 1 / \mathrm{P} 2$ ratio during plateau fell to around 1 and below. Time courses of both amplitudes were strongly correlated with changes in the mean ICP; however, slightly higher correlation was observed for P2 (group-averaged correlation coefficient equal 0.98 [0.93-0.99] vs. 0.95 [0.82-0.96] for P1). The magnitude of decrease in $\mathrm{P} 1 / \mathrm{P} 2$ ratio between baseline and plateau phases was not correlated with either baseline ICP, change in
ICP, or elasticity estimated based on the CSF dynamics model. It showed weak although statistically significant, inverse correlation with baseline $\mathrm{P} 1 / \mathrm{P} 2$ ratio $(R=-0.38, p=0.03$; Fig. $4)$, with largest changes observed in cases where baseline ICP waveform contained $\mathrm{P} 1$ dominating over $\mathrm{P} 2$. Figure 5 shows an example of changes in ICP waveform between baseline and plateau for patients with high and low baseline $\mathrm{P} 1 / \mathrm{P} 2$ ratio.

\section{Comparison of three estimates of cerebrospinal compliance}

Figure 6 shows an illustrative example of the time courses of compliance estimates obtained with each of the three methods for a single patient. Compliance estimates were positively and statistically significantly $(p<0.05)$ correlated: $0.94[0.88-$ 0.97] for $\mathrm{C}_{\mathrm{CSF}}$ vs. $\mathrm{C}_{\mathrm{CaBV}}, 0.77$ [0.63-0.91] for $\mathrm{C}_{\mathrm{CSF}}$ vs. $\mathrm{C}_{\mathrm{P} 1 /}$ ${ }_{\mathrm{P} 2}$, and 0.68 [0.48-0.91] for $\mathrm{C}_{\mathrm{CaBV}}$ vs. $\mathrm{C}_{\mathrm{P} 1 / \mathrm{P} 2}$. Similarly, compliance estimates $\mathrm{C}_{\mathrm{CaBV}}$ and $\mathrm{C}_{\mathrm{P} 1 / \mathrm{P} 2}$ showed inverse correlation with the mean ICP, although the correlation was stronger for $\mathrm{C}_{\mathrm{CaBV}}(-0.82[-0.71--0.86])$ than $\mathrm{C}_{\mathrm{P} 1 / \mathrm{P} 2}(-0.71[-0.46-$ $-0.79]$ ); as $\mathrm{C}_{\mathrm{CSF}}$ was calculated using the mean ICP itself, this pair of parameters was not compared.

Between baseline and plateau phase of the infusion test, group-averaged mean ICP increased from 13.4 [9.5-16.5] to $22.6[18.7-28.0] \mathrm{mm} \mathrm{Hg}$. Accordingly, all three methods showed a statistically significant decrease in compliance estimates between baseline and plateau (Wilcoxon signed rank test $p$-value $<10^{-4}$ ) but with a varying degree of absolute changes, with the largest change observed for the values obtained with the $\mathrm{CaBV}$ model (baseline vs. plateau, 1.16 [0.71$1.75] \mathrm{cm} / \mathrm{mm} \mathrm{Hg}$ vs. $0.72[0.50-1.00] \mathrm{cm} / \mathrm{mm} \mathrm{Hg}$ ), smaller for the CSF dynamics model $(0.67[0.37-1.16] \mathrm{ml} / \mathrm{mm} \mathrm{Hg}$ vs.
Fig. 3 Illustrative example of time courses of amplitude of peaks $\mathrm{P} 1$ and $\mathrm{P} 2$ for a single patient. Full pulse-by-pulse time courses are presented as dots while 30-pulse moving averages are presented as solid lines. a Mean intracranial pressure (ICP). b Amplitude of peaks P1 (light grey symbols) and P2 (dark grey symbols) of the ICP pulse waveform a
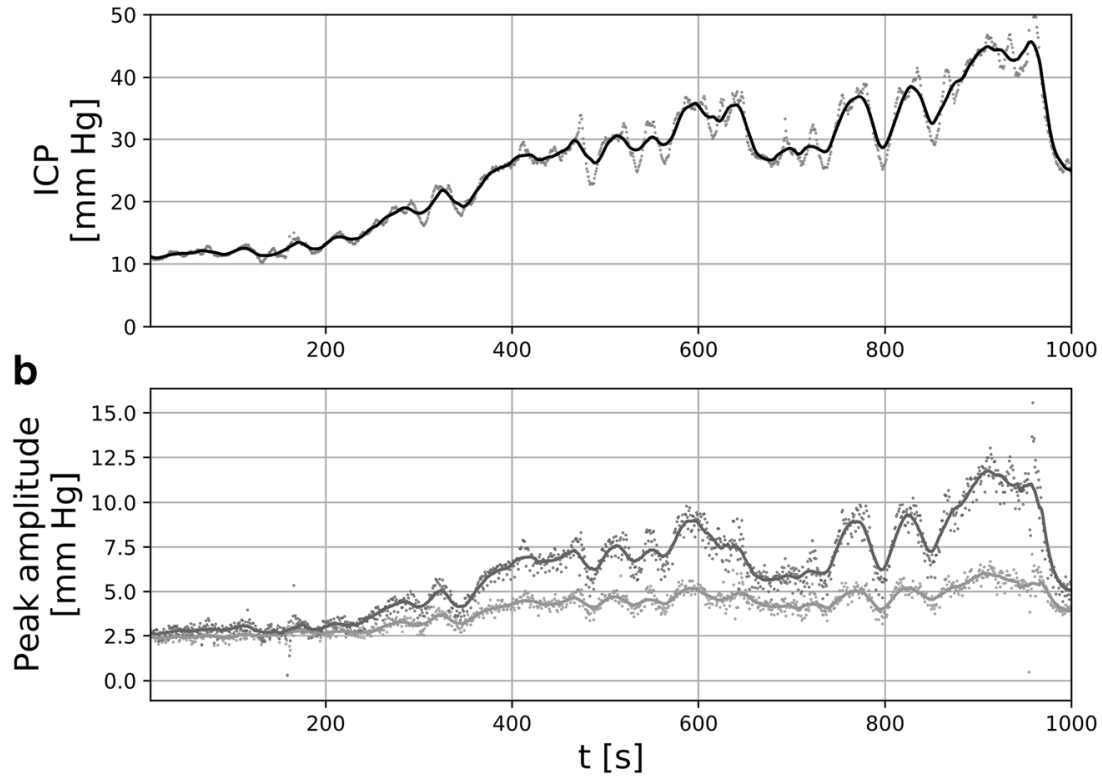


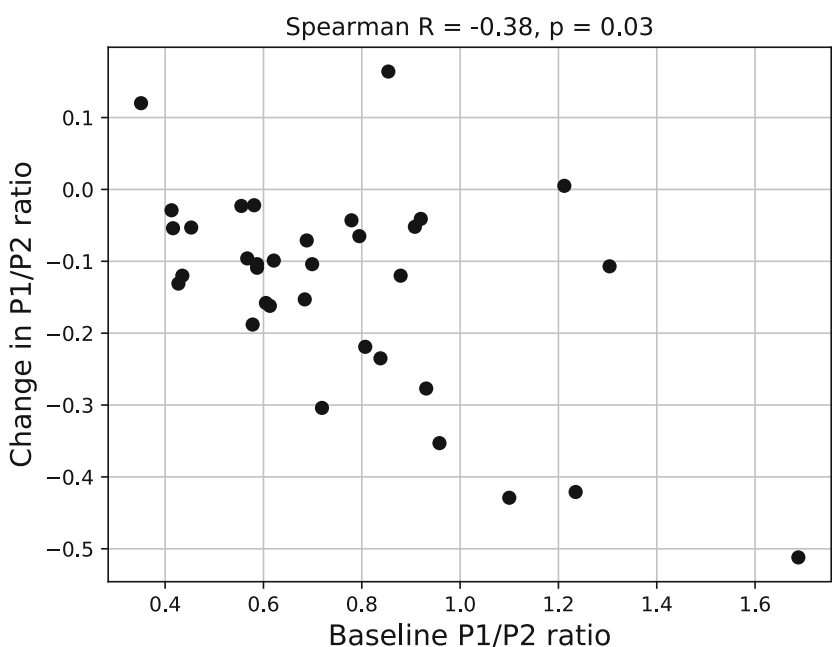

Fig. 4 Relationship between baseline $\mathrm{P} 1 / \mathrm{P} 2$ ratio and change in $\mathrm{P} 1 / \mathrm{P} 2$ ratio between baseline and plateau phases of infusion test. Values above the scatter plot indicate Spearman correlation coefficient and its $p$-value

$0.27[0.17-0.51] \mathrm{ml} / \mathrm{mm} \mathrm{Hg}))$, and smallest for the $\mathrm{P} 1 / \mathrm{P} 2$ ratio (0.69 [0.58-0.90] vs. 0.57 [0.46-0.74]). Decrease in compliance estimates relative to baseline was $46.3 \%$ [36.5-65.1\%], $41.0 \%$ [30.8-48.4\%], and $16.4 \%$ [7.3-27.9\%] for the $\mathrm{C}_{\mathrm{CSF}}$, $\mathrm{C}_{\mathrm{CaBV}}$, and $\mathrm{C}_{\mathrm{P} 1 / \mathrm{P} 2}$ method, respectively.

\section{Discussion}

The analysis of ICP pulse waveform, which relies on the assumption that variations in the pulsatile component of ICP reflect the pressure response to volume changes induced by

a
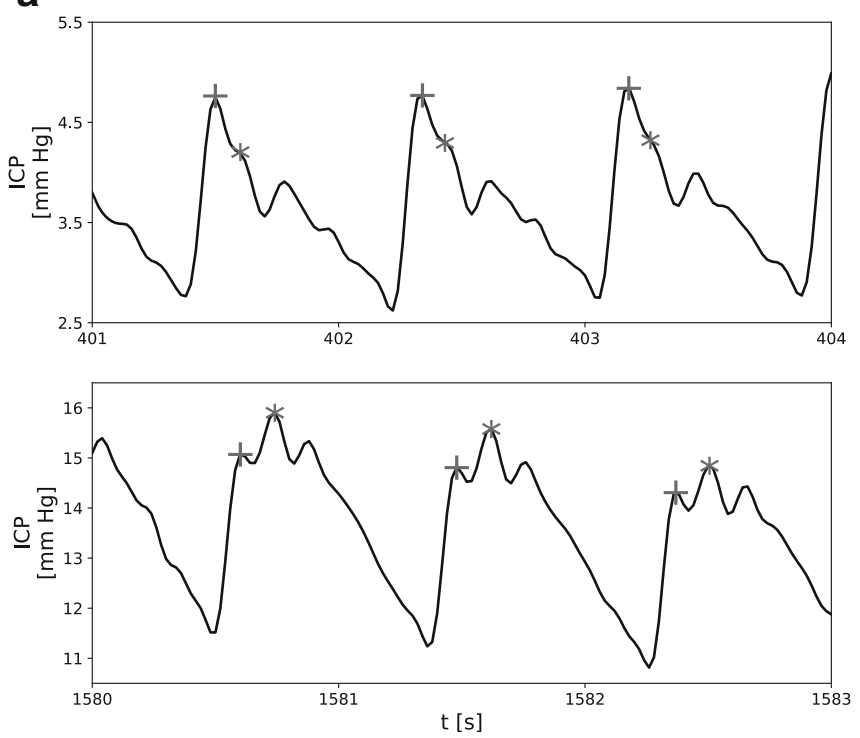

Fig. 5 Illustrative examples of changes in intracranial pressure (ICP) pulse waveform between baseline and plateau phases of infusion test. Baseline and plateau phases are presented in the top and bottom plots, the flow of cerebral blood in each cardiac cycle [2], has been the basis of various indirect methods of compensatory reverse estimation proposed over the years, such as the amplitudepressure curve [31], high-frequency centroid [28], or RAP index [11]. The P1/P2 ratio, although suggested as a measure of cerebral compliance in the 1980 s, so far has not been explored in much detail. A study by Cardoso et al. [6] showed that hyperventilation-induced reduction in the mean ICP is accompanied by a decrease in $\mathrm{P} 2$ and relatively small change in P1, while similar reduction caused by head elevation or CSF withdrawal has little to no effect on the shape of the pulse waveform. It was suggested that this would indicate the dependence of $\mathrm{P} 2$ on cerebral compliance. Results of the present study are in accordance with previously reported observations $[6,22]$. Increase in the mean ICP caused by infusion of volume into the CSF space produced a noticeably larger change in the amplitude of P2 for similar baseline amplitudes of P1 and $\mathrm{P} 2$, resulting in decreases in the $\mathrm{P} 1 / \mathrm{P} 2$ ratio. Individual time courses of the P1/P2 ratio followed the time courses of compliance estimated based on the CSF dynamics model relatively well, with mean correlation coefficient at the level of 0.75 .

The differences in the time courses can be explained by the dependence of $\mathrm{C}_{\mathrm{CSF}}$ on the trend in the mean ICP, used both in the estimation of elasticity and $\mathrm{P}_{0}$ and further calculation of compliance, as the $\mathrm{P} 1 / \mathrm{P} 2$ ratio is calculated only from the shape of the ICP waveform and on a pulse-by-pulse basis. However, this effect was partially mitigated by the use of moving averages in correlation analysis. The slightly weaker correlation with values of $\mathrm{C}_{\mathrm{CaBV}}$ may in turn be the effect of inclusion of another signal, $\mathrm{CBFV}$, in the model. Although the extent of b
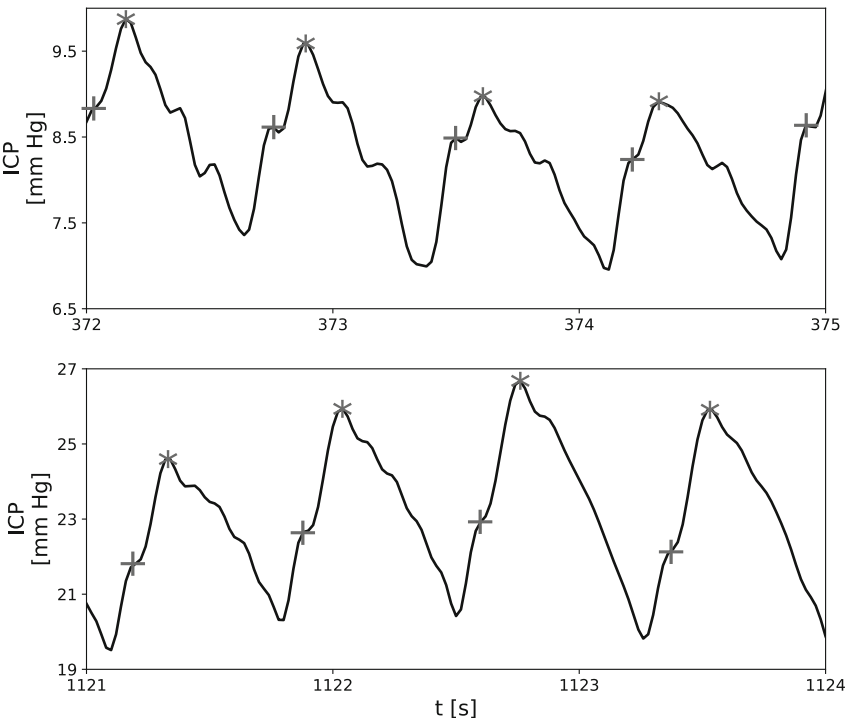

respectively. Location of peaks $\mathrm{P} 1$ and $\mathrm{P} 2$ is indicated by cross (P1) and star (P2) signs. a Patient with high baseline P1/P2 ratio. b Patient with low baseline $\mathrm{P} 1 / \mathrm{P} 2$ ratio 

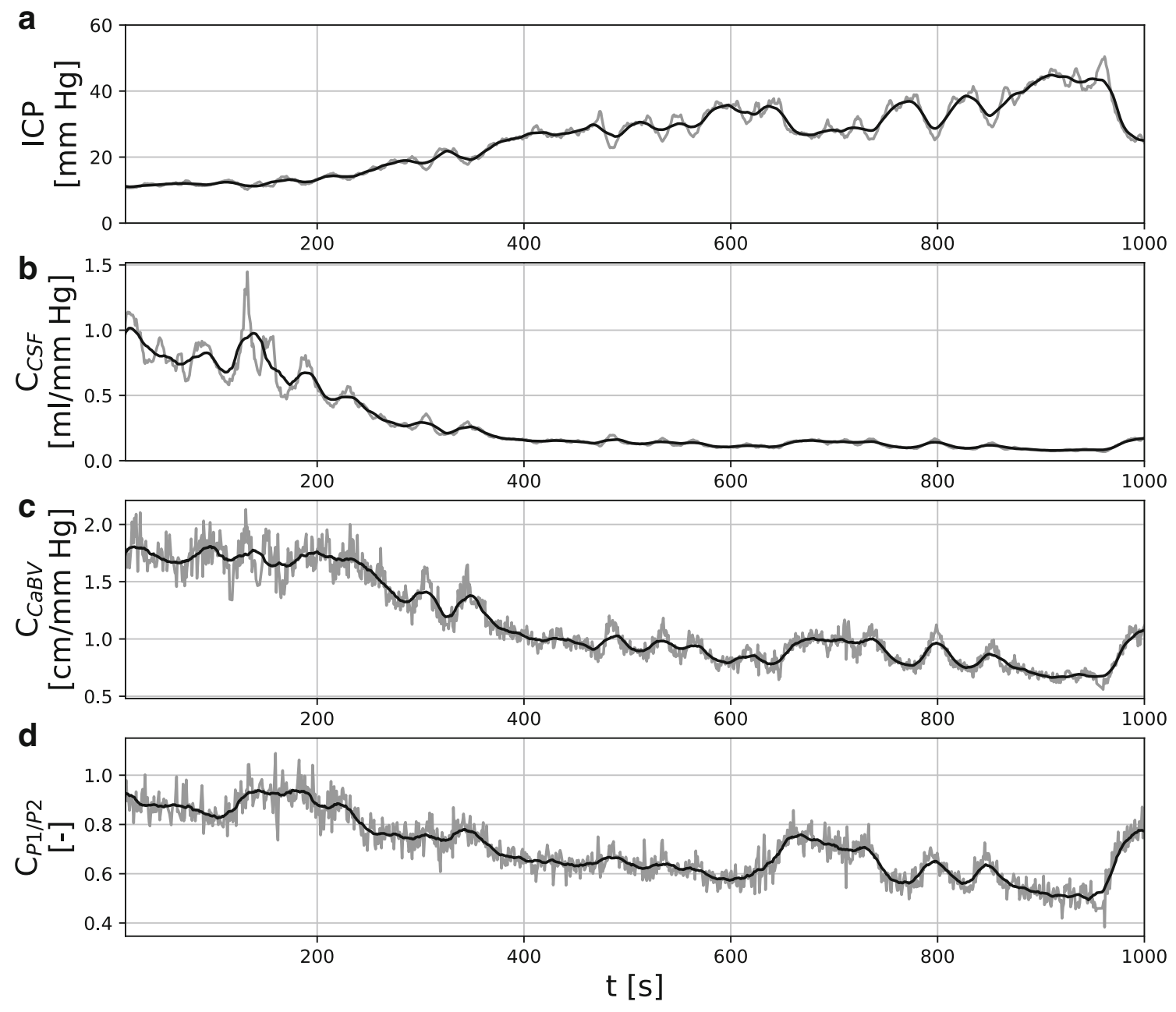

Fig. 6 Illustrative example of time courses of compliance estimates for a single patient. Full pulse-by-pulse time courses are presented as grey lines, while 30-pulse moving averages are presented as black lines. a

decrease in compliance varied between methods, largely due to varying range of values obtained with each approach, all three methods allowed for statistically significant differentiation between the 'high' and 'low' compliance state associated with baseline and plateau phases of the infusion test. However, as the $\mathrm{CaBV}$ and P1/P2 methods examine the ICP pulse waveform resulting from unknown volume load, they are indirect measures that cannot be translated to units of compliance, and straightforward comparison of the values is not feasible. Table 1 outlines major features and limitations of each method of compliance estimation considered in this study.

One advantage of assessing cerebral compliance based on the $\mathrm{P} 1 / \mathrm{P} 2$ ratio is the fact that it does not depend on external volumetric manipulation necessary to derive the pressurevolume curve. In principle, the method also does not require any additional signals beside ICP that are needed to estimate changes in CBV. It should be noted, however, that the major difficulty lies in reliable, fully automated detection of peaks P1 and P2. A number of algorithms of often impressive complexity
Mean intracranial pressure (ICP). b-d Compliance estimates obtained with b CSF dynamics model $\left(\mathrm{C}_{\mathrm{CSF}}\right)$, c cerebral blood volume model $\left(\mathrm{C}_{\mathrm{CaBV}}\right)$ and $\mathbf{d} \mathrm{P} 1 / \mathrm{P} 2$ peak ratio of ICP pulse waveform $\left(\mathrm{C}_{\mathrm{P} 1 / \mathrm{P} 2}\right)$

have been proposed over the years for the task of ICP peak identification $[5,14,17,30]$. Those methods remain a relatively recent development in the continuously advancing field of biomedical signal processing, and despite promising results reported so far, they are yet to find acceptance in the medical community and most importantly validation in large scale trials that would allow for introduction to standard clinical practice. Still, the ICP pulse waveform exhibits large inter- and intrapatient differences, with varying height and prominence of characteristic peaks that may not all be visible even in patients with normal compliance. Whereas the detection of P2 is usually possible despite pathological rounding of the pulse, the detection of P1 becomes unachievable. Incorporation of the ABP signal may improve general peak identification accuracy by allowing for better differentiation between P1 and P2 candidates. Nevertheless, the P1/P2 ratio approach relies heavily on the performance of the underlying detection algorithm, and that in turn requires that the signal is recorded with sufficiently good quality of ICP pulse waveform. 
Table 1 Comparison of three methods of cerebrospinal compliance estimation

\begin{tabular}{llll}
\hline Characteristic & Method & & \\
\cline { 2 - 4 } & $\begin{array}{l}\text { Based on the model of CSF } \\
\text { dynamics }\end{array}$ & $\begin{array}{l}\text { Based on the evaluation of } \\
\text { CaBV from TCD recordings }\end{array}$ & $\begin{array}{l}\text { Based on the analysis of the } \\
\text { P1/P2 ratio of ICP pulse waveform }\end{array}$ \\
\hline Units & $\begin{array}{l}\text { Absolute }[\mathrm{ml} / \mathrm{mm} \mathrm{Hg}] \\
\text { Assessment }\end{array}$ & $\begin{array}{c}\text { Express compliance per unit of } \\
\text { cross-sectional area of insonated } \\
\text { vessel } \\
\text { [cm/mm } \mathrm{Hg}] \\
\text { Continuous but limited by positioning } \\
\text { of TCD probes }\end{array}$ & Relative changes only [dimensionless] \\
Accuracy & $\begin{array}{c}\text { One-off measurement (based } \\
\text { on a recording from entire } \\
\text { infusion test) } \\
\text { Availability }\end{array}$ & $\begin{array}{c}\text { Good } \\
\text { Always when access to CSF space } \\
\text { is possible }\end{array}$ & $\begin{array}{l}\text { Relative changes only } \\
\text { Requires TCD monitoring }\end{array}$ \\
Additional requirements & $\begin{array}{c}\text { Requires invasive ICP measurement } \\
\text { Requires invasive ICP measurement } \\
\text { (with good quality of pulse waveform) }\end{array}$ & $\begin{array}{c}\text { Requires invasive ICP measurement } \\
\text { (with good quality of pulse waveform) }\end{array}$ \\
\hline
\end{tabular}

In the present study, in order to provide as reliable as possible assessment of the feasibility of using the P1/P2 ratio as an estimate of cerebrospinal compliance, P1 and P2 candidates identified by the algorithm using both the analysis of ICP pulse waveform and its relationship with ABP (where available), CBFV, and $\mathrm{CaBV}$ signals were manually reviewed and corrected in cases of insufficient detection accuracy. In order to consider validating this approach in the clinical setting, further work is required to refine the prototype and develop it into a fully automated method of peak identification. Moreover, a comprehensive solution would not only need to be capable of analysing in real time the large variety of ICP pulse waveform shapes encountered in patients with intracranial pathologies but also detecting artefacts and selecting pulses where the peaks, particularly P1, become impossible to distinguish due to the rounding of the waveform and not due to the low quality of the signal.

On the other hand, assuming that the peaks are identified reliably, it is theoretically possible to compare the results between separate measurements and between individuals. A previous study by Fan et al. [15] attempted to use the P2/P1 ratio (as opposed to the $\mathrm{P} 1 / \mathrm{P} 2$ ratio used in the present study) as a predictor of disproportionate increases in ICP in TBI patients. Elevated $\mathrm{P} 2$ amplitude was signified by $\mathrm{P} 2 / \mathrm{P} 1$ ratio equal to or exceeding 0.8 . The study showed that the $\mathrm{P} 2 / \mathrm{P} 1$ ratio, while not a unique predictor of intracranial hypertension episodes, was significantly higher in the group exhibiting ICP increases. A different study in paediatric hydrocephalus patients confirmed the applicability of the $\mathrm{P} 2 / \mathrm{P} 1$ ratio in the identification of intracranial hypertension and assessment of the response to shunting [3]. It should be noted that other works focused on the classification of ICP pulse waveform patterns [14, 26] suggested that decrease in compliance is associated with P2 visibly dominating over $\mathrm{P} 1$. A threshold ratio of 0.8 could therefore potentially lead to the inclusion of normal waveforms in the elevated P2 group and influence the results.
Ultimately, the use of the $\mathrm{P} 1 / \mathrm{P} 2$ ratio as an indicator of decreased buffering capacity of the cerebrospinal system would require further investigation with regard to the correlation between the value of $\mathrm{P} 1 / \mathrm{P} 2$ ratio and the clinical status of the patient, as this measure cannot be directly converted to units of compliance. Given earlier reports on the relationship between measures of compliance and compensatory reserve and outcome in TBI patients [25, 27, 28, 32], combined with the possibility of continuously recording the ICP waveform in modern neuro-critical care units, this avenue of study seems nonetheless to be a promising one.

\section{Limitations}

Compliance estimation based on the evaluation of changes in CBV as used in this study is based on a number of assumptions, including constant diameter of insonated vessels during TCD recording. Due to unknown cross-sectional area of the vessels, $\mathrm{C}_{\mathrm{CaBV}}$ values cannot be calibrated in units of compliance. The same applies to the $\mathrm{P} 1 / \mathrm{P} 2$ ratio as changes in the ICP pulse waveform are analysed as a response to unknown blood stroke volume. Consequently, direct comparison between those two methods and the 'gold standard' approach based on the model of CSF dynamics was limited to the correlation between the time courses.

Moreover, this study was performed as a retrospective analysis of infusion test recordings of the ICP signal with the additional requirement of availability of simultaneously collected CBFV. As TCD measurements are not part of routine clinical investigation in NPH patients, the number of available recordings was limited, and given the size of the study group, the results of this study should be regarded as preliminary. Considered recordings were not collected with the explicit purpose of analysing the ICP pulse waveform in detail, which led to a relatively high 
percentage of cases (almost $30 \%$ of the initial dataset) excluded on the basis of low signal quality.

However, even assuming sufficiently high quality of the signal, the visibility of peaks remains a significant limitation. On the other hand, pulses with indistinguishable P1 due to the rounding of the waveform constitute a separate class of signals. Whereas they do not allow for monitoring of relative changes over time, they could be incorporated as a form of low compliance indicator. This could be a viable solution especially in long-term monitoring aimed at detecting decreases in compensatory reserve, where peak visibility is expected to vary.

\section{Conclusions}

Apart from the 'gold standard' method, compliance of the cerebrospinal fluid system may be evaluated using pulse waveform of ICP and TCD recordings of blood flow velocity in the cerebral arteries. The latter two methods agree with the 'gold standard' approach based on volume addition. It potentially opens new perspectives for continuous brain compliance monitoring in various clinical scenarios.

List of abbreviations ABP, Arterial blood pressure [mm Hg]; CBFV, Cerebral blood flow velocity [cm/s]; CBV, Cerebral blood volume [ml]; $\mathrm{CSF}$, Cerebrospinal fluid; ICP, Intracranial pressure [mm Hg]; NPH, Normal-pressure hydrocephalus; TBI, Traumatic brain injury; TCD, Transcranial Doppler

Funding AK received support from the Polish National Agency for Academic Exchange under the International Academic Partnerships programme. ZC, MC, PS and MP were supported by the European Union INTERREG Programme project REVERT 2020. The funders had no role in the study design, data collection and analysis, decision to publish or preparation of the manuscript.

\section{Declarations}

Ethical standards Data used in this study were collected with approval from the local ethics committee at Addenbrooke's Hospital, Cambridge, UK, and the study adhered to the tenets of the Declaration of Helsinki.

Conflict of interest MC and PS have a financial interest in part of licencing fee of ICM+ software (https://icmplus.neurosurg.cam.ac.uk) used in this study. Other authors declare that they have no conflict of interest.

Open Access This article is licensed under a Creative Commons Attribution 4.0 International License, which permits use, sharing, adaptation, distribution and reproduction in any medium or format, as long as you give appropriate credit to the original author(s) and the source, provide a link to the Creative Commons licence, and indicate if changes were made. The images or other third party material in this article are included in the article's Creative Commons licence, unless indicated otherwise in a credit line to the material. If material is not included in the article's Creative Commons licence and your intended use is not permitted by statutory regulation or exceeds the permitted use, you will need to obtain permission directly from the copyright holder. To view a copy of this licence, visit http://creativecommons.org/licenses/by/4.0/.

\section{References}

1. Alperin N, Sivaramakrishnan A, Lichtor T (2005) Magnetic resonance imaging-based measurements of cerebrospinal fluid and blood flow as indicators of intracranial compliance in patients with Chiari malformation. J Neurosurg 103:46-52. https://doi.org/10. 3171/jns.2005.103.1.0046

2. Ambarki K, Baledent O, Kongolo G, Bouzerar R, Fall S, Meyer ME (2007) A new lumped-parameter model of cerebrospinal hydrodynamics during the cardiac cycle in healthy volunteers. IEEE Trans Biomed Eng 54:483-491. https://doi.org/10.1109/TBME. 2006.890492

3. Ballestero MFM, Frigieri G, Cabella BCT, de Oliveira SM, de Oliveira RS (2017) Prediction of intracranial hypertension through noninvasive intracranial pressure waveform analysis in pediatric hydrocephalus. Childs Nerv Syst 33:1517-1524. https://doi.org/ 10.1007/s00381-017-3475-1

4. Bishop SM, Ercole A (2018) Multi-scale peak and trough detection optimised for periodic and quasi-periodic neuroscience data. Acta Nerochirurgica Suppl 126:189-195. https://doi.org/10.1007/978-3319-65798-1 39

5. Calisto A, Galeano M, Serrano S, Calisto A, Azzerboni B (2013) A new approach for investigating intracranial pressure signal: filtering and morphological features extraction from continuous recording. IEEE Trans Biomed Eng 60:830-837. https://doi.org/10.1109/ TBME.2012.2191550

6. Cardoso ER, Rowan JO, Galbraith S (1983) Analysis of the cerebrospinal fluid pulse wave in intracranial pressure. J Neurosurg 59: 817-821. https://doi.org/10.3171/jns.1983.59.5.0817

7. Cardoso ER, Reddy K, Bose D (1988) Effect of subarachnoid hemorrhage on intracranial pulse waves in cats. J Neurosurg 69:712 718. https://doi.org/10.3171/jns.1988.69.5.0712

8. Carrera E, Kim D-J, Castellani G, Zweifel C, Czosnyka Z, Kasprowicz M, Smielewski P, Pickard JD, Czosnyka M (2010) What shapes pulse amplitude of intracranial pressure? J Neurotrauma 27:317-324. https://doi.org/10.1089/neu.2009.0951

9. Chopp M, Portnoy HD (1980) Systems analysis of intracranial pressure. Comparison with volume-pressure test and CSF-pulse amplitude analysis. J Neurosurg 53:516-527. https://doi.org/10. 3171/jns. 1980.53.4.0516

10. Contant CF, Robertson CS, Crouch J, Gopinath SP, Narayan RK, Grossman RG (1995) Intracranial pressure waveform indices in transient and refractory intracranial hypertension. J Neurosci Methods 57:15-25. https://doi.org/10.1016/0165-0270(94)00106-q

11. Czosnyka M, Guazzo E, Whitehouse M, Smielewski P, Czosnyka Z, Kirkpatrick P, Piechnik S, Pickard JD (1996) Significance of intracranial pressure waveform analysis after head injury. Acta Neurochir 138:531-542. https://doi.org/10.1007/BF01411173

12. Czosnyka M, Czosnyka Z, Momjian S, Pickard JD (2004) Cerebrospinal fluid dynamics. Physiol Meas 25:R51-R76. https:// doi.org/10.1088/0967-3334/25/5/R01

13. Eklund A, Smielewski P, Chambers I, Alperin N, Malm J, Czosnyka M, Marmarou A (2007) Assessment of cerebrospinal fluid outflow resistance. Med Biol Eng Comput 45:719-735. https://doi.org/10.1007/s11517-007-0199-5

14. Elixmann IM, Hansinger J, Goffin C, Antes S, Radermacher K, Leonhardt S (2012) Single pulse analysis of intracranial pressure for a hydrocephalus implant. Conference Proceedings of the IEEE Engineering in Medicine and Biology Society, In, pp 3939-3942

15. Fan J-Y, Kirkness C, Vicini P, Burr R, Mitchell P (2008) Intracranial pressure waveform morphology and intracranial adaptive capacity. Am J Crit Care 17:545-554

16. Germon K (1988) Interpretation of ICP pulse waves to determine intracerebral compliance. J Neurosci Nurs 20:344-351. https://doi. org/10.1097/01376517-198812000-00004 
17. Hu X, Glenn T, Scalzo F, Bergsneider M, Sarkiss C, Vespa P (2010) Intracranial pressure pulse morphological features improved detection of decreased cerebral blood flow. Physiol Meas 31:679695. https://doi.org/10.1088/0967-3334/31/5/006

18. Katzman R, Hussey F (1970) A simple constant-infusion manometric test for measurement of CSF absorption. I. Rationale and Method. Neurology 20:534-544. https://doi.org/10.1212/wnl.20.6. 534

19. Kim D-J, Kasprowicz M, Carrera E, Castellani G, Zweifel C, Lavinio A, Smielewski P, Sutcliffe MPF, Pickard JD, Czosnyka M (2009) The monitoring of relative changes in compartmental compliances of brain. Physiol Meas 30:647-659. https://doi.org/ 10.1088/0967-3334/30/7/009

20. Kim D-J, Czosnyka Z, Kasprowicz M, Smielewski P, Baledent O, Guerguerian A-M, Pickard JD, Czosnyka M (2012) Continuous monitoring of the Monro-Kellie doctrine: is it possible? J Neurotrauma 29:1354-1363. https://doi.org/10.1089/neu.2011. 2018

21. Kirkness CJ, Mitchell PH, Burr RL, March KS, Newell DW (2000) Intracranial pressure waveform analysis: clinical and research implications. J Neurosci Nurs 32:271-277. https://doi.org/10.1097/ 01376517-200010000-00007

22. Kuramoto S, Moritaka K, Hayashi T, Honda E, Shojima T (1986) Non-invasive measurement in intracranial pressure and analysis of the pulse waveform. Neurol Res 8:93-96. https://doi.org/10.1080/ 01616412.1986.11739737

23. Marmarou A, Shulman K, LaMorgese J (1975) Compartmental analysis of compliance and outflow resistance of the cerebrospinal fluid system. J Neurosurg 43:523-534. https://doi.org/10.3171/jns. 1975.43.5.0523

24. Marmarou A, Shulman K, Rosende R (1978) A nonlinear analysis of the cerebrospinal fluid system and intracranial pressure dynamics. J Neurosurg 48:332-344. https://doi.org/10.3171/jns.1978.48. 3.0332

25. Maset AL, Marmarou A, Ward JD, Choi S, Lutz HA, Brooks D, Moulton RJ, DeSalles A, Muizelaar JP, Turner H, Young HF (1987) Pressure-volume index in head injury. J Neurosurg 67: 832-840. https://doi.org/10.3171/jns.1987.67.6.0832

26. Nucci CG, De Bonis P, Mangiola A, Santini P, Sciandrone M, Risi A, Anile C (2016) Intracranial pressure wave morphological classification: automated analysis and clinical validation. Acta Neurochir 158:581-588. https://doi.org/10.1007/s00701-0152672-5

27. Pillai S, Praharaj SS, Rao GSU, Kolluri VRS (2004) Cerebral perfusion pressure management of severe diffuse head injury: effect on brain compliance and intracranial pressure. Neurol India 52:67-71

28. Robertson CS, Narayan RK, Contant CF, Grossman RG, Gokaslan ZL, Pahwa R, Caram P, Bray RS, Sherwood AM (1989) Clinical experience with a continuous monitor of intracranial compliance. $\mathrm{J}$ Neurosurg 71:673-680. https://doi.org/10.3171/jns.1989.71.5. 0673

29. Ryder HW, Espey FF, Kimbell FD, Penka EJ, Rosenauer A, Podolsky B, Evans JP (1953) The mechanism of the change in cerebrospinal fluid pressure following an induced change in the volume of the fluid space. J Lab Clin Med 41:428-435

30. Scalzo F, Asgari S, Kim S, Bergsneider M, Hu X (2012) Bayesian tracking of intracranial pressure signal morphology. Artif Intell Med 54:115-123. https://doi.org/10.1016/j.artmed.2011.08.007

31. Szewczykowski J, Śliwka S, Kunicki A, Dytko P, Korsak-Śliwka J (1977) A fast method of estimating the elastance of the intracranial system. J Neurosurg 47:19-26. https://doi.org/10.3171/jns.1977. 47.1.0019

32. Zeiler FA, Ercole A, Cabeleira M, Beqiri E, Zoerle T, Carbonara M, Stocchetti N, Menon DK, Smielewski P, Czosnyka M, Anke A, Beer R, Bellander BM, Buki A, Chevallard G, Chieregato A, Citerio G, Czeiter E, Depreitere B, Eapen G, Frisvold S, Helbok R, Jankowski S, Kondziella D, Koskinen LO, Meyfroidt G, Moeller K, Nelson D, Piippo-Karjalainen A, Radoi A, Ragauskas A, Raj R, Rhodes J, Rocka S, Rossaint R, Sahuquillo J, Sakowitz O, Stevanovic A, Sundström N, Takala R, Tamosuitis T, Tenovuo O, Vajkoczy P, Vargiolu A, Vilcinis R, Wolf S, Younsi A (2019) Compensatory-reserve-weighted intracranial pressure versus intracranial pressure for outcome association in adult traumatic brain injury: a CENTER-TBI validation study. Acta Neurochir 161: 1275-1284. https://doi.org/10.1007/s00701-019-03915-3

Publisher's note Springer Nature remains neutral with regard to jurisdictional claims in published maps and institutional affiliations. 